\title{
Leiomyosarcoma of vascular origin: case report
}

\author{
Yessica Margarita González-Cantú ${ }^{1}$, Martha Lilia Tena-Suck ${ }^{2}$, Santos Serna-Reyna ${ }^{3}$, Hector \\ Sánchez-Maldonado ${ }^{4}$, Samuel Quintanilla-Garza ${ }^{5}$, Karina Larios-Cárdenas ${ }^{6}$, Alberto García de la \\ Fuente $^{1}$, Cristina-Rodríguez Padilla ${ }^{1}$
}

1. Departamento de Inmunología y Virología, Facultad de Ciencias Biológicas, Universidad Autónoma de Nuevo León (UANL), San Nicolás de los Garza, Nuevo León, México. 2. Departamento de Neuropatología Instituto Nacional de Neurología y Neurocirogía, Ciudad de México. 3. Departamento de Traumatología y Ortopedia, Hospital Christus Muguerza Sur, Monterrey, Nuevo León, México. 4. Departamento de Cirugía, Unidad Médica de Alta Especialidad No.25 del IMSS. Monterrey, Nuevo León, México. 5. Unidad de Medicina Familiar No.73 IMSS, Saltillo, Coahuila, México. 6. Departamento de Anatomía Patológica, Unidad Médica de Alta Especialidad No.25 del IMSS. Monterrey, Nuevo León, México.

Correspondence: Yessica Margarita González-Cantú. Address: Departamento de Inmunología y Virología, Facultad de Ciencias Biológicas, Universidad Autónoma de Nuevo León (UANL), San Nicolás de los Garza, Nuevo León, México. E-mail: ymgc1301@gmail.com

Received: May 28, 2015

DOI : $10.5430 /$ crcp.v2n4p60
Accepted: July 20, 2015

Online Published: September 2, 2015

URL: http://dx.doi.org/10.5430/crcp.v2n4p60

\section{Abstract}

Leiomyosarcomas of vascular origin are uncommon. Originated from the smooth muscles of tunica media of major blood vessels leiomyosarcoma of venous origin is 5 times more common than those of arterial origin, $50 \%$ of cases originated in the lower vena cava. Most cases are presented in women with a median age of 50 years. We reported a 65 -year-old woman, onset of symptoms 3 months before diagnosis with: pain, palpable tumor $(9 \mathrm{~cm} \times 5 \mathrm{~cm})$, edema of the left lower extremity and unilateral intermittent claudication. CT with a vascularized mass, unrelated to adjacent muscle (tibialis posterior) of the left lower pelvic member, it displaces the path of the neurovascular bundle, with a probable origin in the posterior tibial vein. Total tumor resection was performed. Pathology reports a high-grade vascular leiomyosarcoma. T2a, Nx, Mx. Stage IIc. The diagnosis was confirmed by immunohistochemistry. The patient had adequate postoperative recovery without complications. Later the patient received 37 cycles of post-operatory radiotherapy. Currently (July 2015) the patient is asymptomatic. We present this case because although the frequency of this type of tumor is low, should be considered as a differential diagnosis in patients with mass, claudication and pain in lower pelvic member.

\section{Key words}

Vascular leiomyosarcoma, Leiomyosarcoma, Connective and soft tissue neoplasms

\section{I ntroduction}

Sarcoma is a cancer that arises from cells of mesenchymal origin, such as bone, cartilage, muscle, fat, vascular, or hematopoietic tissue. It is a very rare form of cancer with over 50 histologic subtypes ${ }^{[1]}$. Sarcomas are generally classified according to the normal cell line that they most closely look like, which stands up from mesenchymal cell lines. Of all soft tissue sarcomas, around 5\%-10\% are leiomyosarcoma (LMS) ${ }^{[1-4]}$. Sarcomas are rare malignant tumors with a large variety of histologic subtypes, approximately $1 \%$ of adult malignancies ${ }^{[1]}$, for high-risk factor, known by tumor grade, size and location, local control is enhanced with postoperative adjuvant radiation. Local recurrence rates fluctuate, reliant on 
primary site ${ }^{[1,2]}$. It discusses diagnostic evaluation and the principles of management including imaging, biopsy, staging, treatment, follow-up, and the importance of a multidisciplinary approach ${ }^{[1-3]}$. LMS are smooth muscle tumors, can be divided into three types according to their site of presentation: Leiomyosarcomas of soft tissues are the most common presentation, followed by cutaneous leiomyosarcoma, and vascular LMS.

Vascular LMS are a rare tumor and most cases are located in the inferior vena cava and could be presented in any organ and any venous or arterial location ${ }^{[4]}$. Vascular LMS, can affect veins anywhere, mainly affects the leg tributary veins, those tumors that originate in the saphenous vein occurring in $25 \%$, and the rest of veins in $25 \%$ in decreasing order corresponds to the femoral vein, the internal jugular, the iliac, and the popliteal veins ${ }^{[4-6]}$. A correct radiologic diagnosis of vascular LMS could be completed by: CT, MRI, ultrasound (US), and cavography ${ }^{[4]}$. Definitive diagnosis of vascular LMS still needs histological investigation and tissue obtaining is usually done by laparotomy or percutaneous, needle aspiration or biopsy ${ }^{[5-7]}$. Total surgical resection is the best treatment of choice ${ }^{[2]}$. The use of adjuvant therapy for vascular LMS is accepted. The aim of this paper was a case report vascular LMS high grade from the left posterior tibial vein in a 65 years old female.

Figure 1. CTA of the left lower pelvic member with a vascularized mass unrelated to adjacent muscle. It displaces the path of the neurovascular bundle, with a probable origin in the posterior tibial vein.

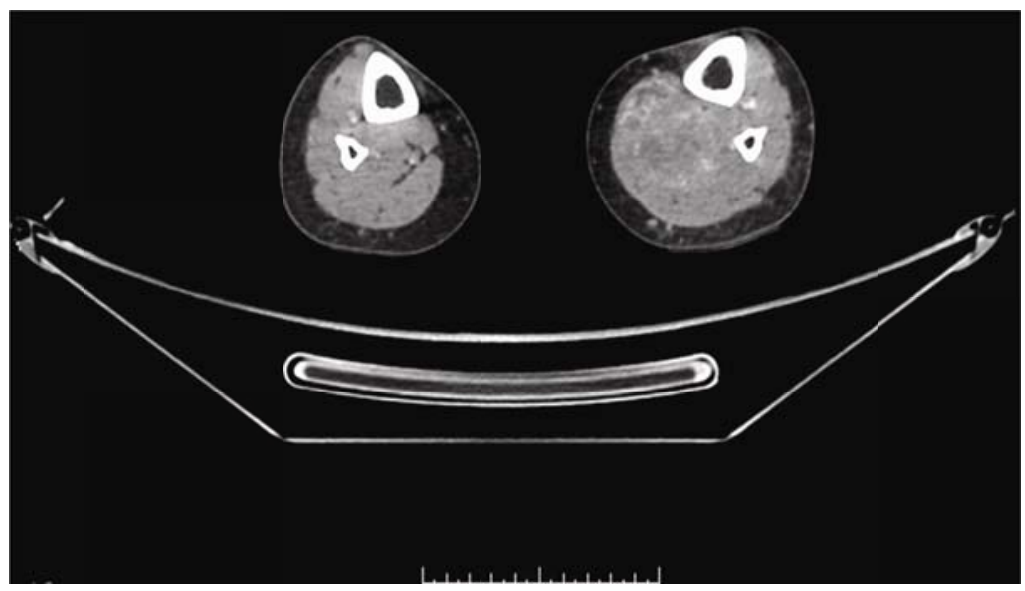

\section{Clinical cases}

A 65-year-old woman without clinical history of vascular disturbance, she presented progressive left leg pain, and unilateral intermittent claudication and rapid growth of the nodular lesion until up to $9 \mathrm{~cm} \times 5 \mathrm{~cm}$ in diameter, this tumor also produced edema of the left lower extremity 3 months before. The CT showed a vascularized mass, unrelated to adjacent muscle (tibialis posterior) of the left lower pelvic member, it displaced the path of the neurovascular bundle, a probable origin in the posterior tibial vein was suspected (see Figure 1). Total tumor resection and tibial vein reconstruction was performed. Gross aspect of the specimen revealed a polypoidal mass measuring $(7.5 \mathrm{~cm} \times 3.5 \mathrm{~cm} \times$ $2.8 \mathrm{~cm}$ ), weight of 75.5 grams. The mass well encapsulated, firm consistency, light brown color with hemorrhagic areas. A cut section it was yellowish, with gelatinous appearance and hemorrhagic focus (see Figure 2A-2B). Microscopic examination revealed a high grade spindle cell malignant tumor composed of diffuse proliferation of large pleomorphic spindly cells with eosinophilic cytoplasm and vesicular nuclei (see Figure 3A, 3B and 3C), with large pleomorphic cells (see Figure 3D and 3E), of necrosis areas were noted and atypical mitotic figures were observed, at less 10-15 per 10 high power fields mitosis were counted (see Figure 3F). Immunohistochemistry revealed that the tumor cells were strong positive immunoreaction to desmin and actin smooth muscle actin (SMA). While calretinin, inhibin, chromogranin, synaptophysin, S100, pancytokeratins, CD34, VIII factor, and CD68 were negative (see Table 1). Based on microscopic and immunohistochemical findings, final diagnosis was of vascular leiomyosarcoma, stage IIC (T2a, Nx, Mx) AJCC. The patient received post-operatory radiotherapy, a total of 37 cycles. The patient has been followed for 3 year after surgery, there was no evidence of tumor recurrence or distant metastasis. 
Figure 2. Gross aspect of the tumor. A. It is light brown and has defined borders. B. section presents a yellowish, gelatinous material and areas with hemorrhage.
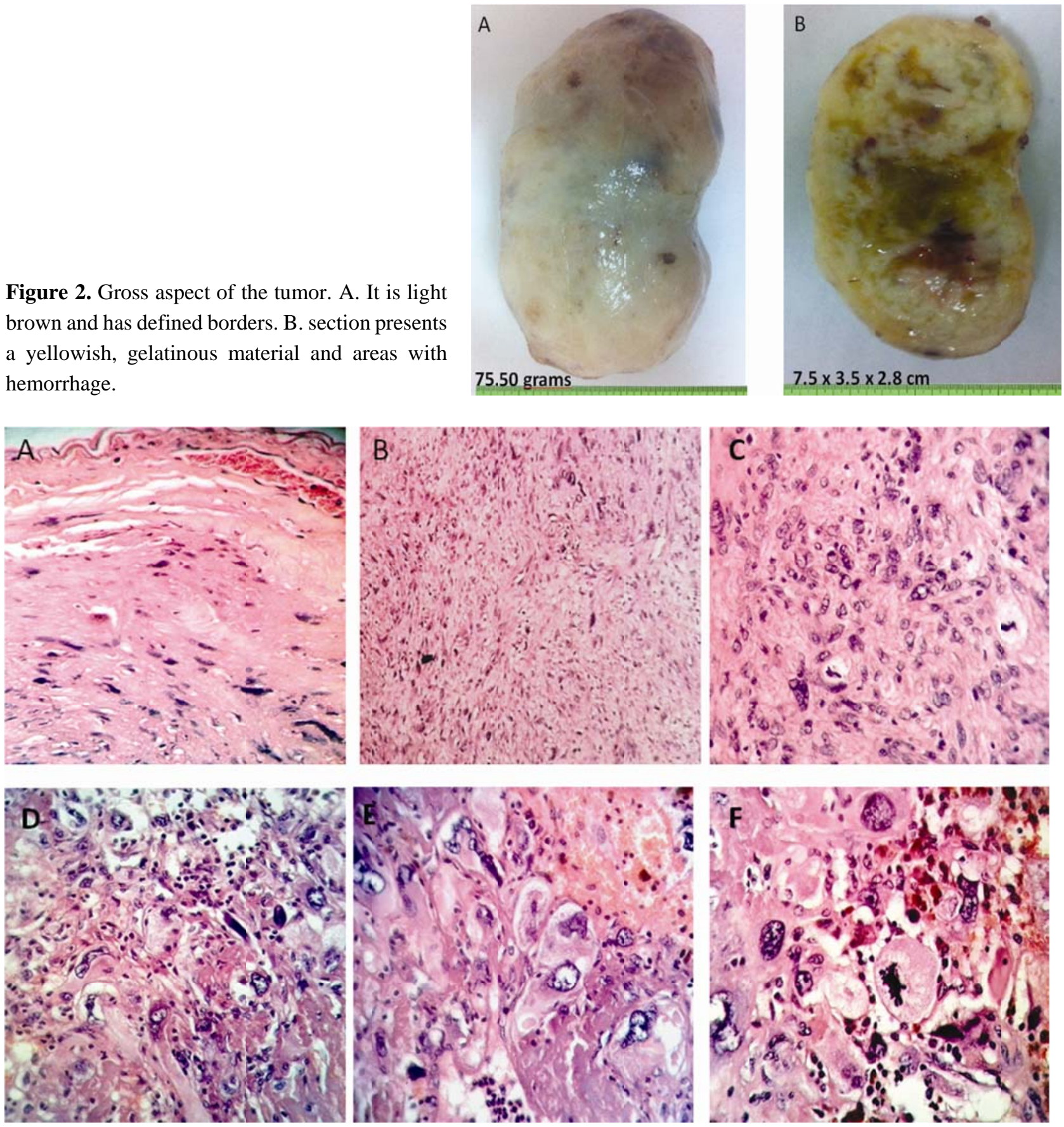

Figure 3. Histopathology. A. Histological image that shows tumoral cells under the vascular endothelium (H\&E×100).

B. The tumoral cells with fusocellular distribution of variable size. C-F. Observed that the neoplastic cells with marked nuclear pleomorphism and atypical mitosis (H\&E×400).

Table 1. Primary antibodies used and their results

\begin{tabular}{lll}
\hline Antibody & Neoplastic cell & Endothelial cell \\
\hline Actin Smooth Muscle & $(+)$ & $(-)$ \\
Desmin & $(+)$ & $(-)$ \\
Factor VIII & $(-)$ & $(+)$ \\
CD34 & $(-)$ & $(+)$ \\
\hline
\end{tabular}




\section{Discussion}

Vascular LMS is a rare tumor origin the media of vessel walls, low extremities veins are the most commonly veins affected, furthermore, the popliteal and tibial veins even less frequently, some few cases have been published ${ }^{[1,4-6]}$. The diagnostic of leiomyosarcoma, it must first be distinguished from other types of tumors, that differentiate these tumors from other soft tissue sarcomas it's not easy to do, a good histological and immunohistochemical study using markers are useful for the diagnosis. The prognosis and treatment varies according to location, preoperational stage and histological type and histological grade of the primary tumor as well as the presence of metastasis at moment of diagnosis. There have been only a few hundred published reports of vascular LMS. This type of lesions blocks the veins by either intraluminal or extra luminal participation or by external pressure, due to tumors growing in the surrounding tissues ${ }^{[4]}$. Abed $\mathrm{R}$ et al. (2009) reported 16/208 cases of vascular LMS (5.8\%), those tumours were straight arising from the blood vessels, tumors reach a height between $3 \mathrm{~cm}-33 \mathrm{~cm}$. Most of them were high grade histologically, and 7/16 patients (44\%) at the time of diagnosis. Dzsinich et al. (1992) ${ }^{[5]}$ reported on 13 cases of vascular LMS, which 8/13 arose from the inferior cava vein 2/13 from the iliac vein, and 2/13 cases from the saphenous vein. Svarvar et al. $(2007)^{[6]}$ reported on 225 patients with LMS of all types from the Scandinavian Sarcoma Group with a cumulative survival of $49 \%$ at 10 years. The tumours in Berlin's series (1984) ${ }^{[8]}$ were located in the superficial femoral vein in 3 patients, in the great saphenous vein in one case, in the popliteal vein in one case, and in the axillary vein in one case. Five cases in that series died of metastatic disease, and only one patient was still alive with metastatic disease within 5 years of treatment ${ }^{[8]}$.

Regarding to the follow-up, the results of Abet series ${ }^{[4]}$ showed a bad outcome in vascular LMS with $75 \%$ of patients died with metastatic disease within the first 3 years of finding, while good local control by surgery and radiotherapy are achieved, while, those results are very similar to the series of Berlin et al. (1984) ${ }^{[8]}$ Leu and Makek (1986) ${ }^{[9]}$ and Hadju et al. (1979) ${ }^{[10]}$.

The clinical manifestation of those tumor and tumor location are: pain with varying degrees of intensity, with gait disturbance, intermittent claudication, to the presence of tumor that can grow quickly causing symptoms of vascular compression, like edema and paresthesia, depending on compression and occlusion of vital structures ${ }^{[7]}$. The involvement of other adjacent structures, like bone, nerves or vascular structures.

The definitive diagnosis of vascular LMS is performed by histological examination and immunohistochemistry techniques ${ }^{[4-8]}$. Macroscopic appearance or gross aspect of the tumor, it usually attains large size with rubbery consistency and soft if necrotic and usually show hemorrhage and bigger tumors often showed cystic structures and myxoid degeneration ${ }^{[3]}$.

The histological examination of LMS is similar at any location. Histological appearance the vascular LMS it is characterized by spindle shaped cells with eosinophilic cytoplasm with muscular striation and cigar shaped rounded nuclei ${ }^{[11]}$. Immunohistochemical staining is helpful for definitive diagnosis, and usually positive immunoreactivity to SMA, vimentin, desmin, calponin, and smooth muscle myosin heavy chains. They also reveal negative immunoreaction for S-100, alfa-inhibin, and CD117 ${ }^{[1]}$. The differential diagnoses of LMS including others sarcomas composed by spindle cells fascicles: fibrosarcoma, synovial sarcoma, rhabdomyosarcoma, inflammatory pseudotumor, neurofibroma and hemangiopericytoma. However, based on the tumor site and size, the prognosis and possible treatments varies.The prognosis factor of patient with vascular LMS depend patient age, size, histologic grade, mitotic activity, and stage of the tumor. Hilliard NJ et al. (2005) ${ }^{[11]}$ proposed to a grading method based on mitotic figures (MF): high grade ( $\geqslant 10 \mathrm{MF} /$ $10 \mathrm{hpfs}$ ); intermediate grade (5-9 MF/10 hpfs); and low grade (1-4 MF/10 hpfs) ${ }^{[11]} 46 \%$ corresponding to high grade, $17 \%$ intermediate grade, and 36\% are low grade. ${ }^{[11]}$ Torjani M et al. (1984) gave emphasis to seven histological criteria: tumor differentiation, cellularity, atypia, pleomorphism, giant cells, mitosis features, necrosis and embolism ${ }^{\text {[12] }}$. While many staging systems exist for soft tissue sarcoma, the most frequently used system is the AJCC staging system is a classification system developed by the American Joint Committee on Cancer ${ }^{[13]}$. It utilizes in part the TNM scoring system: Tumor size, Lymph Nodes affected and Metastases. This categorizes the tumor stranded upon the tumor size, tumor location, as superficial or deep, histologic grade and metastasis. Total surgical resection is the best treatment ${ }^{[13]}$. 
Radiation therapy is an important additional treatment for successful rates of local control when surgical margins are close, especially in high-grade sarcomas ${ }^{[14,15]}$. It is unclear whether chemotherapy would have made a difference in the prognosis of these patients. However in systemic disease, chemotherapy is recommended. Vascular LMS origin has a poor prognosis compared to another soft tissue tumors, could be because these tumours have direct access to the bloodstream, causing more often in distant metastases ${ }^{[16]}$. They are generally poor prognosis tumors.

\section{Conclusions}

We report a case of a vascular LMS originated from the left posterior tibial vein, this tumor is very rare and therefore requires a diagnosis and therapeutic management with multidisciplinary approach.

\section{Acknowledgements}

Thanks to the staff working in the laboratory (Laboratorio de Inmunología y Virología, Facultad de Ciencias Biológicas, Universidad Autónoma de Nuevo León) for academic and economic support.

\section{References}

[1] Weiss SW, Goldblum JR. Leiomyosarcoma in Enzinger and Weiss's Soft Tissue Tumors. 7th edition. St. Louis, Mo, USA: Mosby; 2008.

[2] Röhrborn A, Röher HD. Surgical aspects in the multidisciplinary treatment of soft tissue sarcomas. Praxis (Bern 1994). 1998; 87(34): 1050-60.

[3] Ameeri S, Butany J, Collins MJ, et al. Leiomyosarcoma of the inferior vena cava. Cardiovasc Pathol. 2006; 15: 171-3. PMid: 16697934. http://dx.doi.org/10.1016/j.carpath.2005.08.011

[4] Abed R, Abudu A, Grimer RJ, et al. Leiomyosarcomas of vascular origin in the extremity. Sarcoma. 2009; 2009: 385164. PMid: 19587823. http://dx.doi.org/10.1155/2009/385164

[5] Dzsinich C, Gloviczki P, van Heerden JA, et al. Primary venous leiomyosarcoma: a rare but lethal disease. Journal of Vascular Surgery. 1992; 15(4): 595-603. http://dx.doi.org/10.1016/0741-5214(92)90003-Q

[6] Svarvar C, Böhling T, Berlin Ö, et al. Clinical course of nonvisceral soft tissue leiomyosarcoma in 225 patients from the scandinavian sarcoma group. Cancer. 2007; 109(2): 282-91. PMid: 17154171. http://dx.doi.org/10.1002/cncr.22395

[7] Killoran T, Wells W, Barth R, et al. Leiomyosarcoma of the popliteal vein. Skeletal Radiol. 2003; 32(3): $174-8$. PMid: 12605285. http://dx.doi.org/10.1007/s00256-002-0498-8

[8] Berlin O, Stener B, Kindblom L-G, et al. Leiomyosarcomas of venous origin in the extremities. A correlated clinical, roentgenologic, and morphologic study with diagnostic and surgical implications. Cancer. 1984; 54(10): $2147-2159$. http://dx.doi.org/10.1002/1097-0142(19841115)54:10<2147::AID-CNCR2820541015>3.0.CO;2-9

[9] Leu HJ, Makek M. Intramural venous leiomyosarcomas. Cancer. 1986; 57(7):1395-1400. http://dx.doi.org/10.1002/1097-0142(19860401)57:7<1395::AID-CNCR2820570726>3.0.CO;2-O

[10] Hadju SI. Pathology of Soft Tissue Tumors. Philadelphia, Pa, USA: Lea \& Febiger; 1979.

[11] Hilliard NJ, Heslin MJ, Castro CY. Leiomyosarcoma of the inferior vena cava: three case reports and review of the literature. Ann Diagn Pathol. 2005; 9(5): 259-66. PMid: 16198953. http://dx.doi.org/10.1016/j.anndiagpath.2005.05.001

[12] Trojani M, Contesso G, Coindre JM, et al. Soft-tissue sarcomas of adults: study of pathological prognostic variables and definition of a histopathological grading system. International Journal of Cancer. 1984; 33(1): 37-42. http://dx.doi.org/10.1002/ijc.2910330108

[13] Fremed D, Faries P, Schanzer H, et al. Primary leiomyosarcoma of saphenous vein presenting as deep venous thrombosis. Vascular. 2013; 22(6): 450-3. PMid: 24347138. http://dx.doi.org/10.1177/1708538113516446

[14] Kawai A, Hashizume H, Inoue H, et al. Vascular reconstruction in limb salvage operations for soft tissue tumors of the extremities. Clinical Orthopaedics and Related Research. 1996; 332: 215-22. PMid: 8913166. http://dx.doi.org/10.1097/00003086-199611000-00029

[15] Tilkorn D, Hauser J, Ring A, et al. Leiomyosarcoma of intravascular origin - a rare tumor entity: clinical pathological study of twelve cases. World Journal of Surgical Oncology. 2010; 8(1): 103. PMid: 21092216. http://dx.doi.org/10.1186/1477-7819-8-103

[16] Roy AD, Deka M, Dutta UC. Vascular leiomyosarcoma of thigh - A rare tumour at an unusual site. The Australasian Medical Journal. 2013; 6(10): 520-23. PMid: 24223070. http://dx.doi.org/10.4066/AMJ.2013.1874 